\title{
Development of diode laser pointer- based Michelson interferometer for the measurement of optical parameters
}

\author{
A. Chaudhary
}

A. K. Chaudhary, "Development of diode laser pointer-based Michelson interferometer for the measurement of optical parameters," Proc. SPIE 9664, Ninth International Topical Meeting on Education and Training in Optics and Photonics, 966423 (24 October 2005); doi: 10.1117/12.2207571 and Photonics, 2005, Marseille, France 
Ref ETOP020

\title{
Development of Diode Laser Pointer(LDP) Based Michelson -Interferometer For the Measurement of Optical Parameters
}

\begin{abstract}
A.K. Chaudhary
Abstract
The paper reports the development of an inexpensive laser diode pointer of wavelength $\lambda=630-680 \mathrm{~nm}$ based Michelson interferometer technique for the measurement of optical parameters such as wavelength of the source, thickness of a thin sheets of glass, mica etc. in the Optics Laboratory of the Department of Physics Addis Ababa University, Addis Ababa, Ethiopia. The He-Ne laser source $(\lambda=632.8 \mathrm{~nm})$ in the Michelson interferometer experiment is replaced by small size battery operated LDP of small beam of diameter $=3 \mathrm{~mm}$ with simple modification in the optics system to measure the aforesaid optical parameters
\end{abstract}

\section{Summary}

The He-Ne laser based Michelson interferometer plays very significant role in modern optics laboratories to demonstrate the phenomena of interference based on the division of amplitude of incident laser beam. In addition, it has been employed to measure the wavelength of the source, thickness and refractive index of thin transparent sheets of glass, mica etc.[1,2]. However, it has been observed that many optics laboratories of the developing countries are not having the facility of $\mathrm{He}-\mathrm{Ne}(\lambda=632.8 \mathrm{~nm})$ laser source therefore, the practical application of this experiment could not be demonstrated .

An innovative attempt has been made to show the use of inexpensive small size battery operated laser diode pointer (LDP) which is mainly used for delivering lectures as an ideal replacement of He-Ne laser. The entire arrangement is small in size and can be used any less equipped laboratory for teaching optics.

The use of LDP as a source is restricted due to its small beam diameter $=3 \mathrm{~mm}$, nongaussian out put beam shape (multimode) and small power ( $1 \mathrm{~mW})$. However, these problems could be over come by an introduction of a convex lens in the path of out going interfering beams of Michelson interferometer.

After doing the initial alignment, the convex lens of $f=+20 \mathrm{~cm}$ is inserted between the beam emitted from LDP and the interferometer. This lens causes the beam to be diverge and have a varying phase across the viewing screen. As it is already mentioned that the beam diameter of the LDP is very small therefore, interference pattern formed on the screen is not very much clear. In order to get the clear and well defined fringes , another convex lens $\mathrm{f}=+10 \mathrm{~cm}$ was introduced in the output path of the beams of interferometer. This arrangement provides well defined bright and dark fringes at regular intervals.

First part of the experiment the wavelength $(\lambda)$ of LDP has been measured using Equation.1 [3], by simply counting the number of fringes on the screen and the distance $(\beta)$ traveled by second mirror with the help of micrometer .

$$
\lambda=2 . \beta / n
$$

where $n=$ number of fringes,$\beta=$ is distance for shifting $80-100$ fringes 
In the second part of the experiment a thin plate of glass/mica is introduced in one of the arms of the Michelson interferometer and rotated along the axis,the resultant shift in the fringes are counted. The thickness of the plate " $\mathrm{t}$ " is given by :

$$
\mathrm{t}=\mathrm{n} \lambda / 2[\sqrt{ }\{\mu 2-\operatorname{Sin} 2 \varphi\}-\{\mu+\operatorname{Cos} \varphi\}+1]
$$

Where $\mu$ is the refractive index of the glass/mica sheet and $\varphi$ is the angle of rotation. The process is repeated three to four times for getting best results. The error occurred in the experiment is order of $5-10 \%$.

\section{References}

1. [1] Jenkins and White, Fundamental of Optics, 4th edition, Mc Graw Hill Book Centre. [2] H. Hernandez, A. Juarez, R. Hernandez, Superficies y Vacio 9, 283 (1999)

2. [3]http://cord.org /cm /lept Module 6.htm 